\title{
Establishment and Clinical Application of One-stage Posterior Limited Lesion Clearanceand Internal Fixation Combined With Personalized Drug Therapy System for Brucellosis Spondylitis
}

\section{Xinmin Yang ( $\square$ yxm11200@126.com )}

The First Affiliated Hospital of Hebei North University https://orcid.org/0000-0002-3209-9100

Ye Tian

The First Affiliated Hospital of Hebei North University

Yao Yao

The first affiliated hospital of Hebei North University

\section{Research article}

Keywords: brucellosis, Spondylitis, Limited lesion removal, Short segment spinal fixation, Personalized drug therapy, Clinical efficacy

Posted Date: January 15th, 2021

DOl: https://doi.org/10.21203/rs.3.rs-146670/v1

License: (c) (1) This work is licensed under a Creative Commons Attribution 4.0 International License.

Read Full License 


\section{Abstract}

Objective

To investigate the feasibility and clinical effect of one-stage posterior limited lesion clearance and internal fixation combined with individualized drugs in the treatment of brucidosis spondylitis.

Methods

44 patients who conform to inclusion criteria, including 2 cases thoracolumbar segment, 32 cases lumbar segment and 10 cases lumbosacral segment. Preoperative VAS(Visua Analogue Scales) 5 8,ODI(Oswestry disability index) 51.15 84.36\%, Body Mass Index $\triangle \mathrm{BMI} \otimes$ 16.2 17.5, American Spinal

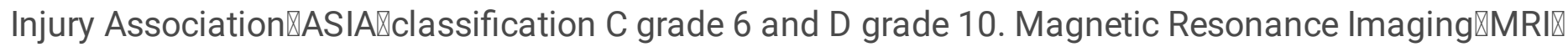
showed compression of dural sac in 16 cases. Preoperative medication regimen was developed according to the patient's medication allergy history, weight, liver and kidney function, and T-lymphocyte spot test $囚 \mathrm{~T}-\mathrm{SOPT} \rrbracket$.

Results

Postoperative individualized medication was used according to the drug sensitivity test and T-SOPT to select 3 kinds of sensitive drugs for 2 courses of treatment, and the patients were clinically cured 3 to 9 months. The evaluation indexes of Erythrocyte Sedimentation Rate $₫ \mathrm{ESR} \rrbracket, \mathrm{C}$-reactive protein $\llbracket \mathrm{CRP} \rrbracket$, Rose Bengal Plate agglutination Test $\triangle R B P T \otimes, V A S, B M I, A S I A, O D I$ and $M R I$ at each time point were significantly improved compared with those before surgery $(P<0.05)$. Follow-up showed that the lesions were completely cleared, inflammation was absorbed, pedicle screw fixation was good, spine stable, intervertebral bone grafting was fused, and the spinal cord were well aligned without compression.

\section{Conclusion}

Personalized medication is highly targeted, conforms to pharmacokinetics, has low toxic and side effects, no drug allergy or drug resistance, so as to reduce medical expenses and obtain the maximum clinical efficacy with the minimum cost. One-stage posterior limited lesion clearance and internal fixation surgery has little trauma, and the lesions are completely removed. The correct choice of screw placement and short segment internal fixation of diseased vertebra is safe and reliable.

\section{Background}

Brucellic disease, it is caused by a zoonotic B infectious disease, also known as wave fever, Mediterranean fever or Maltese fever. It is characterized by long-term fever, hyperhidrosis, joint pain, enlargement of liver, spleen and lymph nodes. In recent years, there have been more than 20 provinces and regions where the outbreak of brucellosis has picked up in China. The disease spread from pastoral to semi-pastoral, agricultural and urban areas. The incidence rate is also on the rise of southern and coastal areas of China. In particular, the incidence rate of city is obviously higher. The epidemic form of 
the disease replaced the large-scale outbreak with multiple, scattered point epidemic[1]. Osteoarthritis is the secondary infection of brucellic disease, the incidence of which is $10 \% \sim 85 \%$. Its most common manifestations are infectious inflammation of the spine and infectious arthritis of the four major joints[2,3]. International scholars call the infectious diseases caused by Brucella invading the intervertebral disc or vertebral body tissue of spine as brucellosis spondylitis[3,4]. The vulnerable sites of brucellosis spondylitis: the first is lumbar vertebrae, the second is thoracic vertebrae and the last is cervical vertebrae. The pathological manifestations of the lesions were mainly discitis, intraspinal granuloma or intraspinal abscess[4-6]. In clinical practice, although through conservative treatment, the majority of patients with spinal brucellosis can be cured. Surgical treatment is necessary when the disc is severely damaged, cauda equina nerve root is damaged, intraspinal abscess is formed, or spinal instability occurs. This study retrospectively analyzed the patients admitted to the First Affiliated Hospital of Hebei North University from January 2014 to January 2019,which used one-stage posterior limited lesion clearance and internal fixation combined with individualized drugs in the treatment of brucellosis spondylitis. This method of treatment has the advantages of small surgical trauma, strong drug pertinence and good therapeutic effect. The report is as follows

\section{Materials And Methods}

\subsection{Inclusion criteria}

\Patients with lumbar single-stage brucellosisspondylitis who have previously received 1 course of regular anti-tuberculosis medication and have no remission of infectious spondylitis $\mathbb{X P a t i e n t s ~ w i t h ~}$ intervertebral disc destruction causing intractable spinal pain $\mathbb{X P a t i e n t s ~ w i t h ~ s i g n i f i c a n t ~ p s o a s ~ a b s c e s s ~ o r ~}$ spinal canal inflammatory granuloma or spinal abscess non-absorptionष区Spinal cord, Cauda equina, or nerve root compression $\mathbb{X S p i n a l}$ stability affected by posterior vertebral column destruction $\mathbb{X}$ follow-up data before and after surgery are complete and not less than 1 year.

\subsection{Exclusion criteria}

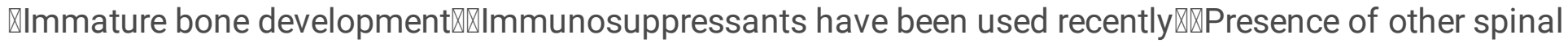
infections other than brucellosis $\mathbb{Q}$ The symptoms of brucellosis are mainly systemic, and the local symptoms of the spine are mild赌The infection only affects the vertebral body and does not involve the

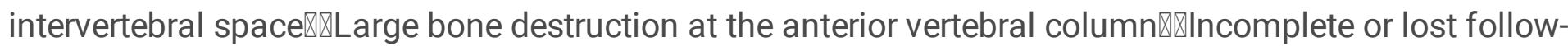
up.

\subsection{General information}

44 patients with brucellosis spondylitis treated in the Department of Orthopaedics, the First Affiliated Hospital of Hebei North University from January 2014 to January 2019 were selected. These cases were diagnosed as confirmed cases[4]. After epidemiological history, clinical manifestations, imaging, and laboratory serology-specific tests (RBPT $\triangle S A T$ ) before admission. There were 26 males and 18 females, aged 32 to 65 years, with an average age of $(42.51 \pm 1.92)$ years. The onset time was 3 to 7 months, with 
an average of $(4.12 \pm 0.23)$ months. Lesions were located in the thoracolumbar segment in 2 cases, lumbar spine in 32 cases, and lumbosacral segment in 10 cases. All patients had a history of fever before surgery, showing an undulant fever of 37.9 to $38.8^{\circ} \mathrm{C}$, with an average of $(38.4 \pm 0.4)^{\circ} \mathrm{C}$. Patients have varying degrees of lower back pain, muscle stiffness, restricted rotation or flexion and extension, the preoperative VAS was $5-8$, with an average (6.56 \pm 1.33$)$, and the ODI was $51.15-84.36 \%$, with an average $(68.84 \pm 4.55) \% .32$ patients with mild to moderate malnutrition, anemia, and hypoproteinemia, BMI was 16.2 to 17.5 , average ( $16.82 \pm 0.24) .16$ cases had symptoms of nerve injury, 6 cases of ASIA neurological grade $C, 10$ cases of $D$ grade. MRI showed 44 cases of intervertebral disc destruction, including 16 cases of dural sac compression. RBPT and SAT were positive in 44 patients. The preoperative ESR was 31 to $105 \mathrm{~mm} / \mathrm{h}$, with an average of $(55.42 \pm 11.42) \mathrm{mm} / \mathrm{h}$; the CRP was 22 to $89 \mathrm{mg} / \mathrm{L}$, with an average of $(55.40 \pm 17.56) \mathrm{mg} / \mathrm{L} .4$ patients were allergic to 2 kinds of first-line anti brucellosis drugs, and 2 patients were intolerant to 1 kind of drugs after operation. Last follow-up for at least 12 months. At the request of the patients, 32 patients underwent secondary surgery to remove spinal internal fixation at an average of $(24.02 \pm 1.22)$ months after surgery.

This clinical study was authorized and approved by the Ethics Committee of the First Affiliated Hospital of Hebei North University (batch number: 2013-10), and informed the patients and their families of the clinical diagnosis and treatment plan, and signed the informed consent of the treatment plan.

\subsection{Treatment method}

\subsubsection{Preoperative preparation and personalized medication plan After admission, patients are} encouraged to rest in bed and implement health education about brucellosis spondylitis to improve medication compliance. X-ray, Computed Tomography $\otimes \mathrm{CT} \otimes$, and MRI examinations are performed to determine the degree of destruction of the

disc, whether there are inflammatory granulomas or abscesses in the spinal canal, whether there is compression in the spinal cord, horsetail, or nerve roots, whether the pedicle bone structure is intact, and whether the soft tissue around the spine is involved. Perfect T-SOPT. According to individual needs, patients need to strengthen systemic nutritional support before surgery, correct malnutrition indicators such as low body weight, hypoalbuminemia, and anemia, and effectively control internal medical diseases such as heart, lung, brain, liver, kidney, endocrine, and blood. Before the operation, individualized anti-brucellosis medications were administered, using a combination, appropriate amount, regular and full-course administration. For confirmed cases, doxycycline + rifampicin + sulfamethoxazole is the first choice[7]. The dose of the drug is based on the weight, liver and kidney function of the patient. Doxycycline (2.2mg/kg once daily, maximum dose $0.2 \mathrm{~g} / \mathrm{kg}$ once daily, for 56 days continuously); Rifampicin ( $10 \mathrm{mg} / \mathrm{kg}$ once daily, maximum dose $0.6 \mathrm{mg} / \mathrm{kg}$ once daily, for 56 days continuously); Sulfamethoxazole composite ( $1.0 \mathrm{~g}$ doubled for the first time, twice a day, for 56 days continuously). TSPOT was used to detect rifampicin resistance. For those who were allergic or intolerant to a rifampicin drug, it could be changed to doxycycline + sulfamethoxazole + streptomycin according to individual allergies and physical conditions, Streptomycin $(20 \mathrm{mg} / \mathrm{kg}$, maximum dose $1.0 \mathrm{~g} / \mathrm{kg}$, intramuscular 
injection, once a day for 21 days). For those who were allergic or intolerant to the two drugs of doxycycline and streptomycin, they can be changed to rifampicin + fluoroquinolones + tobramycin according to the "Brucellosis Diagnosis and Treatment Guide" [8], Tobramycin (1 mg / kg per day, once per 8 hours, the maximum dose is $5 \mathrm{mg} / \mathrm{kg}$ per day, 1 course of treatment on 10-14 days); Patient's liver and kidney function and drug side effects were regularly monitored during medication. Through individualized drug treatment, surgery could be performed only when nutrition was improved, various indicators were stable, and ESR was decreasing, and no higher than $40 \mathrm{~mm} / \mathrm{h}$. [9 10]

\subsubsection{One-stage posterior approach for limited lesion removal and internal fixation The patient was given} general anesthesia, prone position, and a midline incision was taken from the back of the spine. Expose the spinous process, lamina, articular process of the diseased vertebra and one vertebra adjacent to the diseased vertebra, and accurately insert pedicle screws under C-arm. According to the preoperative imaging evaluation, if the pedicle and the upper part of the vertebral body are intact, the lower part of the vertebral body is slightly damaged and the sclerotic bone is less, the pedicle screw can be inserted into the diseased vertebral body after applying streptomycin; If the vertebral body is severely damaged or unstable, the adjacent vertebral body can be selected for pedicle screw fixation across the diseased vertebral body. According to the location of the lesion, part of the facet joints on one or both sides of the diseased intervertebral space were excised, and the dural capsule and the nerve roots of the diseased segment were exposed and separated. Abscesses and granulomas in the spinal canal were removed, and the bone destruction area behind the vertebral body and the infected intervertebral disc tissue were thoroughly scraped until the normal vertebral bone surface leaked. After repeated rinsing, an appropriate amount of the autologous sacrum, the previously cut articular process and a little bite of the lamina were added to $1 \mathrm{~g}$ of streptomycin powder for intervertebral bone grafting. The gelatin sponge was filled behind the bone grafting gap to prevent Bone fragments fall into the spinal canal. According to the preoperative imaging location, if there was a large paravertebral abscess, the abscess could be removed through the transverse process of the diseased vertebra, and then the streptomycin powder could be placed after repeated washing with gentamicin saline. The fixation rod was placed, the screws were pressed and fixed, the scoliosis deformity was corrected, the drainage tube was placed and sutured layer by layer. The operation time was 70 to $120 \mathrm{~min}$, with an average of $(80.15 \pm 9.51) \mathrm{min}$, and the bleeding volume was 200 to $400 \mathrm{~mL}$, with an average of $(250.24 \pm 20.12) \mathrm{mL}$. Bacterial culture, drug sensitivity and pathological examination were carried out on the diseased tissue or pus removed by operation to provide basis for pathological diagnosis and further development of sensitive chemotherapy plan.

\subsubsection{Postoperative management and individualized medication plan After the operation, we continued to} carry out health education and medication compliance guidance, continued to give nutritional support therapy, corrected anemia and hypoproteinemia, stabilized internal diseases, and regularly reexamined blood routine and related biochemical indicators. The drainage tube was removed 48 hours later. The next day, the patient was asked to gradually get out of bed and move with spinal brace. The wearing time was at least 3 months. According to the individualized anti brucellosis medication plan before operation, the anti-brucellosis drugs should be taken on the second day after operation. According to the results of bacterial culture and drug sensitivity, sensitive and non-allergic anti- brucellosis drugs were used, and 
three sensitive drugs were combined for two courses. If there are less than 3 first-line sensitive drugs in the Brucellosis Treatment Guideline, use quinolones or aminoglycosides until the RBPT turns negative, and continue to take the medicine for 2 weeks. During the treatment period, check the liver and kidney functions monthly and find out if there is any abnormal response treatment. When there are less than 3 first-line sensitive drugs in the Brucellosis Treatment Guidelines, use quinolone or aminoglycoside drugs, until RBPT becomes negative for 2 weeks. During the period of medication, the liver and kidney functions were rechecked every month, and the corresponding treatment was carried out in time if any abnormality was found.

\subsection{Follow-up and efficacy evaluation}

Patients came to our hospital for follow-up visits every 3 months after surgery. ESR, CRP and RBPT were used to evaluate the control of inflammation; VAS score was used to evaluate the relief of lumbocrural pain; ODI was used to evaluate the impact of lumbocrural pain on daily life function; ASIA neurological classification was used to evaluate the recovery of spinal cord function; BMI was used to evaluate the nutritional status of patients. All patients underwent imaging evaluation, the stability of spine, fusion of bone graft and fixation of pedicle screw were evaluated by X-ray. 16 patients with ponytail or nerve root injury before operation were examined by MRI to evaluate spinal cord, ponytail or nerves. Root decompression situation. MRI was performed in 16 patients with cauda equina or nerve root injury before operation to evaluate the decompression of spinal cord, cauda equina or nerve root. Standard of bone graft fusion [11]: no tenderness, throbbing pain, or abnormal activity in the diseased spinal segment, and internal fixation is stable; imaging shows that the bone graft particles in the intervertebral space do not fall off or be absorbed, the intervertebral space is fuzzy and there is bone trabecula passing through, no pseudojoint formation, and bone remodeling is obvious.

\subsection{Statistical methods}

Statistical Product and Service Solutions (SPSS) 21.0 statistical software was used to analyze the data. The data of ESR and CRP were expressed by mean \pm standard deviation. VAS score, ODI score, Asia grade and BMI were tested by independent sample t-test, the test standard was $p<0.05$.

\section{Results}

\subsection{The general situation of postoperative follow-up of the treatment of one-stage posterior limited lesion clearance and internal fixation combined with individualized drugs.}

In 44 cases of this group, the pathological examination of the diseased tissue removed during the operation was consistent with the histologic manifestation of brucellosis[5]. Among the samples submitted for examination during the operation, 26 cases showed positive bacterial culture results. Results of drug sensitivity test by disk diffusion method: the sensitivity rates of tetracycline, doxycycline, streptomycin, gentamicin, amikacin, tobramycin, ciprofloxacin, ofloxacin and chloramphenicol were all over $90 \%$; The sensitivity rate of rifampicin and compound Xinnuo is about $70 \%$; The drug resistance rate 
to azithromycin, clarithromycin and isoniazid was about $80 \%$. After T-SOPT, 5 cases were resistant to rifampicin. There were no complications such as injury of blood-vessel, nerve and spinal cord during operation. All wounds healing were first intention, without infection or sinus formation or recurrence case. The patients had good mental state, appetite and medication compliance. According to the results of postoperative drug allergy test and T-SOPT, three kinds of sensitive, non-resistant and non-allergic drugs were selected. 2 courses in total ( 56 days as a course). Monitoring the damage of liver and kidney function. Patients with internal diseases before operation and stable disease control after operation. In this group, 44 patients reached the clinical cure standard at 3 9 months after operation, with an average of $(4.56+1.31)$ months[12]. There are 28 patients, $18-26$ months postoperatively, with an average of $(24.02 \pm 1.22)$ months, Surgical removal of spinal internal fixation and during the operation, the bone tissue of the vertebral body was taken through the nail channel and sent to the pathological examination, no inflammatory manifestation was found.

\subsection{Inflammatory indexes and VAS, ODI scores before and after the treatment of one-stage posterior limited lesion clearance and internal fixation combined with individualized drugs.}

After the treatment of one-stage posterior limited lesion clearance and internal fixation combined with individualized drugs. The results of ESR, C-reactive protein, RBPT, VAS and ODI were compared with those before operation, which ESR, C-reactive protein, VAS, ODI scores gradually decreased and The number of patients with RBPT positive decreased gradually. The difference was statistically significant $(P<0.05)$. See Table 1.

Table 1:ESR, C-reactive protein, RBPT and VAS, ODI scores before and after treatment of one-stage posterior limited lesion clearance and internal fixation combined with individualized drugs $\square \overline{\boldsymbol{x}} \pm s \square$

\begin{tabular}{|lllcll|}
\hline & $\mathrm{ESR}(\mathrm{mm} / \mathrm{h})$ & C-reactive protein $(\mathrm{mg} / \mathrm{L})$ & $\mathrm{RBPT}(+)$ & VAS & ODI(\%) \\
\hline Before operation & $55.42 \pm 11.42$ & $55.40 \pm 17.56$ & 44 & $6.56 \pm 1.33$ & $68.84 \pm 4.55$ \\
\hline After3months & $10.11 \pm 1.24$ & $9.17 \pm 1.12$ & 6 & $1.42 \pm 0.52$ & $13.67 \pm 1.33$ \\
\hline After6months & $8.05 \pm 1.47$ & $6.24 \pm 1.34$ & 1 & $1.04 \pm 0.15$ & $2.11 \pm 1.07$ \\
\hline After9months & $6.48 \pm 1.28$ & $5.45 \pm 1.26$ & 0 & 0 & 0 \\
\hline After12months & $5.71 \pm 1.13$ & $4.64 \pm 1.15$ & 0 & 0 & 0 \\
\hline & & & & & \\
\hline
\end{tabular}

Note: comparison with patients before operation. $(P<0.05)$.

\subsection{ASIA result of the treatment of one-stage posterior limited lesion clearance and internal fixation combined with individualized drugs.}

Thear are 16 patients with symptoms of nerve injury were improved after one-stage posterior limited lesion clearance and internal fixation combined with individualized drugs.6 patients At the last follow-up, 
which before operation the result of ASIA was grade C, improved to grade D in 1 case, Grade E in 5 cases. Before treatment, 10 cases were grade $\mathrm{D}$, all of them improved to grade $\mathrm{E}$. The other 28 patients had no symptoms of nerve injury before and after treatment. See Table 2.

Table 2:ASIA grade before and after treatment of one-stage posterior limited lesion clearance and internal fixation combined with individualized drugs.

\begin{tabular}{|llllll|}
\hline \multirow{2}{*}{ Before operation ASIA gread } & \multicolumn{5}{c|}{ After operation ASIA grade } \\
\cline { 2 - 6 } & A & B & C & D & E \\
\hline A & - & - & - & - & - \\
\hline B & - & - & - & - & - \\
\hline C & - & - & - & 1 & 5 \\
\hline D & - & - & - & 0 & 10 \\
\hline E & - & - & - & - & 28 \\
\hline
\end{tabular}

\subsection{BMI evaluation results of nutritional status before and after the treatment of one-stage posterior limited lesion clearance and internal fixation combined with individualized drugs.}

There were 32 patients with mild to moderate malnutrition, anemia and hypoproteinemia before the treatment of one-stage posterior limited lesion clearance and internal fixation combined with individualized drugs, BMI 16.2-17.5, mean (16.82 \pm 0.24$)$. after the treatment, the nutritional indicators were gradually corrected. At the last follow-up, All patients had no anemia, hypoproteinemia or

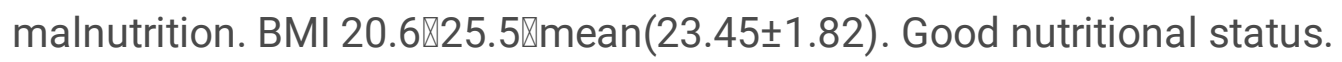

\subsection{Imaging evaluation results of the treatment of one-stage posterior limited lesion clearance and internal fixation combined with individualized drugs.}

After the treatment of one-stage posterior limited lesion clearance and internal fixation combined with individualized drugs. Regular follow-up X-ray examination shows that pedicle screw fixation in both diseased vertebra and normal vertebra showed good control force, no loosening or breaking, stable spine, no loss or absorption of intervertebral bone graft particles, and the fusion time of bone graft was 3-9 months. Average $(4.56 \pm 1.31)$ months. 16 patients were found with cauda equina or nerve root injury before operation. after operation, the MRI results of these patients show that no inflammatory manifestations in the vertebral body of the original lesion, repair of bone damage lesions, complete clearance of paravertebral and intraspinal abscesses and inflammatory granulation tissue or necrotic intervertebral discs, orderly alignment of spinal cord or cauda equina nerves, stable spine. No adjacent vertebral degeneration was found at the last follow-up. 


\section{Discussion}

\subsection{The necessity of the establishment of the system of one-stage posterior limited lesion clearance and internal fixation combined with individualized drug therapy for brucellosis spondylitis}

Brucellosis spondylitis is a specific infection of the human spine infected with brucellosis, which leads to disc inflammatory lesions, vertebra destruction and abscess formation[13,14]. Anti-brucellosis treatment is the most effective and basic method to control brucellosis spondylitis. For the early treatment cases, mainly systemic symptoms, and local spondylitis symptoms mild, imaging manifestations only vertebral body and disc inflammatory changes, but stable spine, no or small bone destruction foci, abscess, no symptoms of nerve injury, most can be cured by conservative treatment $\triangle 7,15 \rrbracket$. It is observed in clinical application that drug resistance rate increases gradually due to non-standard drug treatment in recent years. Tetracycline plus streptomycin, or drug replacement based on this regimen for the treatment of brucellosis recommended in the World Health Organization (WHO) joint bulletin, has an effective rate of only $60 \%$, Liver and kidney function damage is greater, the long-term effect is not good, and the recurrence rate is high $[7,16,17]$. How to improve the cure rate of brucella spondylitis, reduce the recurrence rate, reduce brucella resistance and drug toxicity, to avoid liver and kidney function damage, therefore, the establishment of personalized drug treatment system is very necessary. After standardized conservative treatment, the systemic condition is improved, but the symptoms caused by infectious spondylitis are not improved, and there are obvious intractable low back pain, parvertebral abscess, spinal cord or cauda equina nerve root injury and spinal instability need to be operated on $[4,10,12]$. There are two widely accepted surgical methods at home and abroad, namely posterior or anterior and posterior approaches combined with primary focus removal, bone grafting and spinal internal fixation[18-22]. No matter what kind of operation exist the following disadvantages: large incision, wide exposure area, remove range is too big, vertebral canal expanded decompression and spinal long segmental fixation, operation time is long, traumatic big, more bleeding, length of hospital stay long, slow recovery, the high cost of health care.The incidence of adjacent vertebra disease, screw breakage and rod breakage was high, In particular, combined anterior and posterior surgery is more difficult, and the anatomy is more complicated, intraoperative need changing positions high incidence of infection[18,23]. Therefore, on the basis of relieving pain, clearing lesions and stabilizing the spine, how to reduce surgical trauma, reduce blood loss, shorten hospital stay, accelerate surgical rehabilitation and reduce medical costs is the current trend of surgical development. With the improvement of the level of comprehensive surgical treatment of brucellosis spondylitis, the one-stage posterior limited lesion removal internal fixation combined with individualized drug therapy system can achieve this, so it is very necessary to establish this treatment system.

\subsection{Advantages of one-stage posterior limited lesion clearance for brucellosis spondylitis}

Lesion removal spinal fixation is a classic surgical method in the treatment of brucellosis spondylitis. It can completely remove the lesion, alleviate pain and improve nerve dysfunction, correct scoliosis and restore spinal stability[24]. Complete clearance of the lesion is achieved by extensive decompression of 
the lamina and extensive exposure of the surgical field, and the lesion is removed from the normal tissue. These include paraspinal abscesses and intraspinal inflammatory granulation tissue, abscesses, necrotic intervertebral discs, and damaged cartilaginous surfaces. It has a good effect on controlling the development of the lesion and relieve pain and reduce complications. This operation is performed at the cost of destroying and sacrificing normal tissue to enlarge the wound, and it lacks objective criteria for complete lesion remova. With individualized sensitive drugs pertinence treatment $\mathbb{Z}$ most brucidosis spondylitis can be cured by conservative treatment $[4,7]$.Therefore, from the perspective of promoting the recovery of focal infection, the removal of focal disease is no longer the sole objective of surgical treatment.The main objective of surgical treatment shifted from focusing on the extensive removal of lesions to focusing on the removal of large abscesses that were difficult to absorb and the removal of intraspinal compression of the nervous system to the restoration of spinal stability and the promotion of early recovery[23-25].Postoperative recurrence of brucellosis spondylitis is related to the destruction of spinal stability, and the reconstruction of spinal stability is the key to the success of surgical treatment of infectious spondylitis[10-12,18,19,24].In the research progress of brucellosis spondylitis, domestic and foreign scholars such as Ulu-Kilic A $[14,26,27]$ elaborated that the main limitations of brucellosis spondylitis abscess were distributed in the paraspinal and spinal canal, and the main clinical manifestation was nerve injury caused by spinal cord cauda equina compression. The purpose of clearing the lesion and restoring the nerve function could be achieved through windowing surgery.In this study, the principle of lesion removal was to remove paraspinal difficult to absorb abscess and intraspinal granulation tissue, intervertebral disc, relieve nerve compression and spinal fixation by limited exposure with less trauma. One-stage posterior approach for limited lesion removal and internal fixation surgery was performed. The lamina was not removed after limited exposure through the posterior approach, but epidural abscesses, inflammatory granulation tissue and necrotic intervertebral discs that compressed the spinal cord or cauda equina nerves were removed only by opening a window, through the transverse process the paraspinal abscess was cleared and intervertebral or articular process bone graft was performed. The diseased vertebra or across the diseased vertebra is translocated into the short segment of pedicle screw for fixation[14].The one-stage posterior limited lesion removal and internal fixation can achieve the expected purpose of surgical treatment without damaging the stability of the spine. Compared with the classical operation, it has less trauma, shorter operation time, less intraoperative bleeding, shorter hospital stay and faster recovery.Short segment fixation maximizes spinal mobility and reduces the risk of vertebral degeneration in adjacent segments[11,28].

\subsection{Advantages of individualized drug therapy system for brucellosis spondylitis}

The use of the same treatment regimen for different individuals not only fails to improve the treatment effect, but also results in the waste of medical resources and even harm to patients. At present, the relationship between individual therapy and curative effect has been paid more and more attention by doctors and scholars. On the basis of fully considering the condition, age, sex, constitution, nutritional status, duration of disease, imaging assessment, physiological and pathological characteristics of accompanying internal diseases, other drugs being taken and liver and kidney functions of each patient. According to the individual situation of brucellosis spondylitis, the most suitable drug therapy, which is 
safe, reasonable, effective and economical, and has specific and operable treatment plan, is formulated. The purpose of individualized medication is to improve the efficacy of drugs, reduce the toxic and side effects of drugs, reduce the medical expenses and let patients spend the shortest time and the least money to achieve the best therapeutic effect. Clinicians should not only follow the clinical evidencebased medicine basis, according to the current treatment guidelines to develop the treatment program, but also to specific problems specific analysis of individual treatment, in order to achieve a better clinical effect. Popularized as the bacterial culture and drug susceptibility test as well as to the rifampicin resistant T-SOPT detection technology, implementation of individualized drug therapy in patients with clinical diagnosis of brucella spondylitis. According to the treatment guidelines of brucellosis, three classical anti-brucellosis drugs were selected with tetracycline group as the first, namely doxycycline + rifampicin + sulfamethoxazole.personalized medicine are administered according to individual difference. The use of rifampicin was determined based on T-SOPT, and the dose was given according to the patient's own kg body mass and liver and kidney function. According to pharmacokinetics, the time and times of administration should be determined to prevent poor efficacy due to insufficient dose, or toxic side effects of liver, kidney, gastrointestinal tract and nervous system caused by excessive dose or allergy. Intraoperative inflammatory tissues and abscesses were collected for bacterial culture and drug sensitivity. The postoperative drug treatment plan was adjusted according to the drug sensitivity results, and the highly sensitive drugs were selected in preference, so as to achieve pertinence and operable individualized drug treatment, thus ensuring the treatment effect.

\subsection{The feasibility and efficacy of one stage limited lesion clearance and internal fixation combined with individualized drug therapy in the brucellosis spondylitis}

Through the preoperative and postoperative inflammation control, pain relief, the impact of daily life function, spinal nerve function recovery, nutrition status and imaging findings of comparative analysis. After treatment, inflammatory control indexes including erythrocyte sedimentation rate, c-reactive protein and RBPT in the 44 patients in this group reached normal with time, indicating that the application of this treatment system to cure brucellosis spondylitis is feasible and has a good effect (see Table 1).Through pain relief and improvement of daily life function, it can be known that limited lesion removal can effectively remove inflammatory tissues from the lesion and relieve pain, and posterior spinal internal fixation can immediately restore spinal stability. According to the postoperative VAS ODI score of this group of patients (see Table 1), a good effect can be achieved within a short time, indicating that this treatment system is feasible and plays a good role in alleviating pain and promoting the recovery of daily life function. The ASIA grading evaluation of the postoperative spinal nerve function recovery in this group showed that one-stage posterior approach for limited lesion removal and internal fixation was feasible to remove the lamina and articular process bone, remove the lesion, scrape the abscess and relieve the spinal nerve compression. At the last follow-up, all the 16 patients with symptoms of nerve injury before surgery were effectively improved, including 1 case of ASIA grade D and 15 cases of ASIA grade $\mathrm{E}$ (see Table 2). According to the preoperative and postoperative BMI evaluation of the nutritional status, the preoperative BMI of 32 patients with mild and moderate malnutrition was 16.2 17.5, and the nutritional indicators were gradually corrected after standardized treatment according to the treatment 
system. At the last follow-up, all the patients had a good nutritional status, BMI 20.6 25.5, indicating that this treatment system had a good feasibility and efficacy in improving the nutritional status of brucidosis spondylitis patients. According to the imaging evaluation, both in the diseased vertebral body and in the normal vertebral body, short-segment pedicle screw fixation showed good control force, stable spine and intervertebral bone graft fusion, and no adjacent vertebral degeneration was found, indicating that shortsegment pedicle screw fixation was feasible and effective. With the passage of time, MRI showed the inflammatory absorption of the original lesion vertebral body, Remove completely of perivertebral and intraspinal abscess, inflammatory granulation tissue or necrotic intervertebral disc, and no compression of spinal cord or cauda equina nerves, further indicating that limited lesion clearance was feasible and effective. Spinal internal fixator was removed in 28 patients with primary lesion vertebral body screw placement 18 to 26 months after surgery. During the operation, the vertebral bone tissue was taken through the nail channel and histological examination showed no inflammatory manifestations, It also indicates that the screw placement of the diseased vertebral body is feasible. All the patients in this group showed no damage in liver and kidney function monitoring during individualized drug therapy and all reached the clinical treatment standard. Clinical practice showed that the application of the system as a whole for the treatment of brucidosis spondylitis was feasible and achieved good efficacy.

\section{Abbreviations}

Visua Analogue Scales VAS

Body Mass Index BMI

American Spinal Injury Association ASIA

T-lymphocyte spot test T-SOPT

Magnetic Resonance Imaging MRI

Erythrocyte Sedimentation Rate ESR

Oswestry Disability Index ODI

Rose Bengal Plate agglutination Test RBPT

C-reactive protein CRP

Standard tube Agglutination Test SAT

Computed Tomography CT

Statistical Product and Service Solutions SPSS

World Health Organization WHO

Page 12/15 


\section{Declarations}

\section{Disclosure}

Not applicable

\section{Consent for publication}

Not applicable.

\section{Availability of data and materials}

The datasets used and analysed during the current study are available from the corresponding author on reasonable request.

\section{Competing interests}

The authors declare that they have no competing interests.

\section{Funding}

The study was supported by Key project of health technology research and achievement transformation in Hebei Province (zh2018014) and Tracking items of applicable medical technology in Hebei Province (G2018074).

\section{Authors' contributions}

Xinming Yang designed this study and wrote the manuscript, Tian Ye, Yao Yao collected the data, analyzed and interpreted the patient data. All authors read and approved the final manuscript.

\section{Acknowledgements}

Not applicable

\section{References}

1. Shi Y J, Lai S J, Chen Q L, et al. Analysis on the epidemiological features of human brucellosis in northern and southern areas of China, 2015-2016[J]. Chinese Journal of Epidemiology. 2017, 38(4):435-440.

2. Köse ŞK,Serin SS,Akkoçlu G,etal. Clinical manifestations, complications, and treatment of brucellosis: evaluation of 72 cases.[J].Turkish journal of medical sciences,2014,44(2).

3. Yin $Y L$, Yang $X M$, Tian Y, et al. Clinical epidemiological characteristics, diagnosis and treatment of patients with brucellosis spondylitis. [J]. Chinese Journal of Nosocomiology. 2019,29(18):2820-2824. 
4. Yang X M, Meng X Y, Hu C B, et al. Diagnostic and surgical treatment of Brucella spondylitis. Chinese Journal Bone and Joint Surgery. [J]. 2016,9(04):308-316.

5. Yang X M, Meng XY, Wei S, et al. The comparison of the manifestation of the clinical imageology and pathology between the brucellar spondylitis and the spine turberculosis [J]. Surg Sci, 2014, 5(2): 6069.

6. Bagheri A B,Ahmadi K,Chokan N M J,etal.The Diagnostic Value of MRI in Brucella Spondylitis With Comparison to Clinical and Laborator Findings.[J].Acta Inform Med, 2016,24(2):107-110.

7. Yang X M,Shi W, Meng X Y,et al.The Assessment of the Clinical Effect of the Drug Compatibility and Course of Treatment to the Brucellar Spondylitis[J].Surgical Science,2013,4(1):92-99.

8. Ministry of Health of the PRC. Guidelines for diagnosis and treatment of brucellosis (Trial). [J]. Infectious disease information. 2012ख25(6): 323-324.

9. Yang XM,Shi W, Du Y K,et al. Clinical characteristics and surgical treatment effect of Brucellar spondylitis. [J]. Chinese Journal of Endemiology. 2019, 38(4): 320-324.

10. Tian Ye®Yang Xinming.The Surgical Treatment of Lumbar Brucellar Spondylitis Byposterior Approach. [J].Spine Research $₫ 2018 \otimes 4(1: 3): 1-6$.

11. Sun C F, Yang XM. Safety evaluation and curative effect analysis of disease vertebral nailing and short-segment pedicle internal fixation for brucellar spondylitis of the lumbar vertebrae. [J].Chinese Journal of Injury Repair and Wound Healing. 2017, 12(5): 350-356.

12. Yang $X$ M, Meng $X Y$, Zhang $Y$, et al. Treatment of the brucellar spondylitis in the thoracic and lumbar vertebrae by surgery. [J]. Chinese Journal of Spine and Spinal Cord. 2012邓22(7)『600-606.

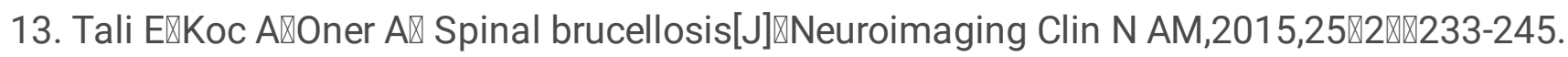

14. Yang $X M$, Wei $S$, Wang $X Y$, et al. Imaging classification and ClinicalSignificance of brucellosis spondylitis[J].J Spine, 2014, 3(3): 172-177.

15. Yao Yao, Yang Xinming. The Senile Brucellosis Spondylitis Clinical Diagnosis and Treatment on as Evaluation Were Retrospectively Analyzed.Surgical Science,2018区9(8):262-280.

16. Saltoglu N, Tasova Y, Inal AS, et al. Efficacy of rifampicin plus doxycycline versus rifampicin plus quinolone in the treatment of brucellosis [J]. Saudi Med J, 2002, 23(8): 921-924.

17. Solera J, Geijo P, Largo J, et al. A randomized, double-blind study to assess the optimal duration of doxycycline treatment for human brucellosis. [J]. Clin Infect Dis, 2004, 39(12): 1776-1782.

18. Yang X M, Zhang L, Jia Y L, et al. Retrospective analysis of posterior or anterior debridement with internal fixation for lumbar brucellosis spondylitis. [J]. Chinese Journal of Anatomy and Clinics. 2015,20(5):428-434.

19. Yang $X M$ M, Zhang L, Zhang $Y$, et al. clinical evaluation of one-stage radical debridement combined with posterior pedicle internal fixation for brucellar spondylitis of the thoracic and lumbar vertebrae. [J]Chinese Journal of Reparative and Reconstructive Surgery. 2012ه26(3):266-271》

20. Pina MA,Modrego PJ,Uroz JJ, et al.Brucellar spinal epidural abscess of cervical location:report of four cases. [J].Eur Neurol,2001,45(4):249-253. 
21. Atonis P,Tzermiadianos M, Gikas A,etal.Surgical treatment of spinal brucellosis. [J].Clin Orthop Relat Res,2006,444(3):66-72.

22. Figen K, Hakki M G, Aysegul S, et al. Brucellar spondylodiscitis: comparison of patients with andwithout abscesses. [J].Rheumatol Int (2013) 33:985-992.

23. Yang $X$ M, Jia Y L. Risk factors for postoperative recurrence of brucella spondylitis. [J]. Chinese Journal Bone and Joint Surgery. 2018,11(9):677-682.

24. Yang X M, Zuo X H, Jia Y L. comparison of effectiveness between two surgical methods in treatment of thoracolumbar brucella spondylitis. [J]. Chinese Journal of Reparative and Reconstructive Surgery. 2014囚28(10):1241-1247.

25. Meng $X Y$,Yang $X \mathrm{M}$, Zhang P, et al. Surgical outcomes of 38 cases of Brucellar spondylitis with nervous lesion. [J]. Orthopedic Journal of China. 2015,23(5):393-401.

26. Ulu-Kilic A『Karakas A『Erdem H囚et al.Update on treatment options for spinal brucellosis[J].Clin Microbiol Infect.2014,20囚2囚:75-82.

27. Turan B,Mustafa K,Hasan I,etal.Clinical manifestations and complications in 1028 cases of brucellosis: A retro spective evaluation and review of the Literature. [J].Int $\mathrm{J}$ Infect Dis. 2010,14(6):e469-478.

28. Hu C B, Yang X M. One stage posterior lumbar radical debridement combined with disease of vertebral nailing and posterior short-segment pedicle internal fixation, and bone grafting on single gap brucellar spondylitis of the lumbar vertebrae. [J]. Chinese Journal of Anatomy and Clinics. 2018,23(2):110-116. 